\title{
Long-term population effect of male circumcision in generalised HIV epidemics in sub-Saharan Africa
}

\author{
Michel Garenne \\ Institut Pasteur, Unité d’Epidémiologie des Maladies Emergentes, 25 Rue du Docteur Roux, 75724 Paris Cedex 15, France \\ e-mail: mgarenne@pasteur.fr
}

\begin{abstract}
This paper examines the complex relationship between male circumcision and HIV prevalence and incidence in sub-Saharan African countries that have generalised epidemics. In South Africa, the mean yearly HIV incidence and an estimate of the net reproduction rate of the epidemic $\left(R_{0}\right)$ (in this case, the ratio of the number of HIV-infected persons between 1994 and 2004 to the number of persons infected in 1994 from which they were presumed to have become infected) were computed from antenatal clinic data for the period 1994-2004, and then compared, by province, to prevailing levels of male circumcision (high, medium and low). In South Africa, mean yearly HIV incidence and net reproduction rate of the epidemic were not lower in provinces with higher levels of male circumcision. For thirteen other countries where Demographic and Health Survey data were available, male HIV prevalence in circumcised and non-circumcised groups was compared. A meta-analysis of that data, contrasting male HIV seroprevalence according to circumcision status, showed no difference between the two groups (combined risk ratio $[R R]=0.99,95 \% \mathrm{Cl}=0.94-1.05)$. Individual case study analysis of eight of those countries showed no significant difference in seroprevalence in circumcised and uncircumcised groups, while two countries (Kenya and Uganda) showed lower HIV prevalence among circumcised groups, and three countries (Cameroon, Lesotho and Malawi) showed higher HIV prevalence among circumcised groups. In most countries with a complex ethnic fabric, the relationship between men's circumcision status and HIV seroprevalence was not straightforward, with the exception of the Luo in Kenya and a few groups in Uganda. These observations put into question the potential long-term effect of voluntary circumcision programmes in countries with generalised HIV epidemics.
\end{abstract}

Keywords: demography, epidemiology, HIV infection, household surveys, meta-analysis, prevention and control, quantitative data, statistical analysis

\section{Introduction}

Numerous studies have shown a relationship between male circumcision and HIV infection. Fink (1986) referred to frequent observations that herpes and syphilis were more common among uncircumcised men, arguing that this could be true as well for cases of HIV infection, in particular because the inner foreskin hosts a large number of Langerhans cells which are prime targets for HIV. Fink (1987) noted that HIV first spread in areas of Africa where most men were not circumcised. Bongaarts, Reining, Way \& Conant (1989) systematically reviewed African ethnic groups practicing circumcision and found a geographical correspondence with areas of high HIV seroprevalence, and a statistical relationship between the proportion of circumcised males and male HIV prevalence in capital cities of 28 countries. Since then, similar observations at the individual level were made in case-control studies as well as in cohort studies. Moses, Bradley, Nagelkerke, Ronald, NdinyaAchola \& Plummer (1990) and Moses, Plummer, Bradley, Ndinya-Achola, Nagelkerke \& Ronald (1994) reviewed 30 epidemiological studies and concluded that a majority showed a significant association between male circumcision and HIV infection. Weiss, Quigley \& Hayes (2000) reviewed 27 studies and, after a meta-analysis, concluded that male circumcision reduces the rate of female-to-male HIV transmission by about half, on average.

These ecological and observational studies were recently confirmed by three randomised controlled trials, conducted in South Africa, Kenya and Uganda, which found that circumcised men had a 51-60\% lower risk of HIV infection over a 12- to 24-month period than did uncircumcised men (Auvert, Taljaard, Lagarde, Sobngwi-Tambekou, Sitta \& Puren, 2005; Bailey, Moses, Parker, Agot, MacLean, Krieger et al., 2007; Gray, Kigozi, Serwadda, Makumbi, Watya, Nalugoda et al., 2007).

There seems little doubt that male circumcision reduces the individual risk of female-to-male transmission of HIV, other factors controlled for. However, how this observation may translate into the long-term population dynamics of HIV epidemics remains an open question. In particular, does a $50 \%$ reduction in annual risk at the individual level translate into a strong reduction of HIV incidence and prevalence at the population level in a generalised HIV epidemic?

Several mathematical models have attempted to predict the possible effect of male circumcision (e.g. see Gray, Li, Wawer, Gange, Serwadda, Sewankambo et al., 2003; Williams, Lloyd-Smith, Gouws, Hankins, Getz, Hargrove 
et al., 2006; Nagelkerke, Moses, De Vlas \& Bailey, 2007; Orroth, Freeman, Bakker, Buvé, Glynn, Boily et al., 2007). However, these exercises do not guarantee prediction of the final impact in populations, especially in situations where the current dynamics of an HIV epidemic remain poorly understood. Indeed, no mathematical model can accurately reproduce observed rates of HIV seroprevalence with realistic parameters in the case of heterosexual transmission.

Several African countries with large groups of circumcised and uncircumcised men allow one to study the differential dynamics of HIV epidemics according to males' circumcision status. Furthermore, the case of countries with minority populations, either circumcised or not, also sheds some light on these complex relationships. These contrasting situations in generalised epidemics are unique, as they provide information of what could be the potential impact of voluntary circumcision programmes. This study uses two types of data for a quantitative investigation: data from antenatal clinics and data from population-based surveys. Since the two methodologies are different, they are presented sequentially.

\section{Data and methods}

The first type of data, used only for South Africa, was the routine surveillance of HIV prevalence of pregnant women at antenatal clinics (sentinel sites). Routine surveillance in South Africa started in 1990 and has been maintained ever since. These data allow one to study the dynamics of the HIV epidemic over time, focusing on women of reproductive age. South Africa is affected by high levels of HIV seroprevalence. For example, HIV seroprevalence among attendants at antenatal clinics in South Africa reached 29.5\% in 2004 (South African Department of Health, HIV and AIDS statistics, available online: www.avert.org/safricastats.htm).

Two population-based studies, published by the Human Sciences Research Council (HSRC) (2002 and 2005), also found high HIV seroprevalence: estimates for 2002 and 2005 , respectively, were $12.8 \%$ and $13.3 \%$ prevalence among females of all ages; $9.5 \%$ and $9.3 \%$ prevalence among men of all ages; and peaks at $32 \%$ and $33 \%$ prevalence for women aged 25-29. These figures were consistent with the antenatal clinic data.

South Africa is divided into nine provinces, and has nine large ethnic groups and four racial groups. With respect to the black African population, some of the provinces are largely ethnic-specific, others (in particular industrialised and urbanised areas) are a mixture of several ethnic groups. Among the major African ethnic groups, three are known to widely practice male circumcision: the Xhosa (who comprise $83 \%$ of the population of the Eastern Cape Province), the Pedi (52\% of the population of Limpopo Province), and the Venda (16\% of Limpopo). Three ethnic groups practice circumcision moderately: Sotho, Ndebele and Shangaan; and three groups practice circumcision rarely: Zulu (comprising $81 \%$ of KwaZulu-Natal Province, and $26 \%$ of Mpumalanga Province), Tswana (65\% of the North West Province) and Swazi (31\% of Mpumalanga). Although never universal or forbidden in any ethnic group, circumcision levels are considered to be about $75 \%$ in the first group (Xhosa, Pedi, Venda), around $45 \%$ in the second group (Sotho, Ndebele, Shangaan), and around $15 \%$ in the third group (Zulu, Tswana, Swazi) (HSRC, 2002). The 2002 HSRC study found that $35 \%$ of men overall in South Africa were circumcised, which is consistent with the proportion expected if projecting from the ethnic composition. A study (RADAR, 2002) conducted among the Pedi in southern Limpopo Province found that $89 \%$ of males aged 14-35 were circumcised. In that study, however, a greater proportion of circumcised men were HIV infected $(7.5 \%$ seroprevalence) than were uncircumcised men (5\% seroprevalence) (pers. comm., Dr Paul Pronik, director of the RADAR project, November, 2006).

Since 1994, HIV seroprevalence data for attendants at antenatal clinics in South Africa have been tabulated and published according to province, by the South African Ministry of Health, the sole consistent series of such data available in Africa. Data for HIV seroprevalence in $1994\left(P_{1994}\right)$ and in $2004\left(P_{2004}\right)$ allow one to reconstruct the dynamics of the epidemics in the provinces over the ten-year-period. Two indices were calculated, these were: the mean yearly HIV incidence, calculated as $\left(P_{2004}-P_{1994}\right) / 10$; and an estimate of the net reproduction rate of the epidemic, calculated as $R_{0}=\left(P_{2004} \bullet K-P_{1994}\right) / P_{1994}$, where $\mathrm{K}$ is the coefficient of population growth between 1994 and 2004. The $\mathrm{K}$ coefficient was calculated for the female adult population aged 15-49 from data provided by Statistics South Africa (available online: www.statssa. gov.za). $R_{0}$ gives the ratio of the number of HIV-infected persons between 1994 and 2004 to the number of persons infected in 1994 from which they were presumed to have become infected. This assumes a 10-year survival rate for HIV-infected persons, and ignores those who became infected and died during the interval, and thus it is obviously an underestimation of the true value. However, the idea is not to provide the best estimate of $R_{0}$, but simply to give a simple measure of the reproductive capacity of the epidemic that can be compared among groups.

Demographic and Health Surveys (DHS) are largescale standardised surveys conducted in many countries on representative samples of the population. Data from 13 surveys conducted in sub-Saharan Africa provided information on both male circumcision status and on HIV seroprevalence in the adult male population (aged 15-54 years). These surveys allow one to compute the ratio of HIV prevalence after many years in the epidemic according to male's circumcision status. The surveys are of high quality and are free from selection biases otherwise built into the antenatal clinic data. They are probably the most reliable source of information currently available on the relationship between HIV seroprevalence and level of male circumcision in national populations. The risk ratio (RR) was calculated as the ratio of HIV seroprevalence among circumcised males to that of uncircumcised males. Statistical testing of the differences was done using standard tests for risk ratios. A combined RR was calculated by meta-analysis, with weights for each survey being proportional to the inverse of the standard error.

\section{Results}

\section{South Africa}

In South Africa, the dynamics of the HIV epidemics in the 
nine provinces differed from what was expected based on the proportions of circumcised men in each province (Table 1). For the country as a whole, mean yearly HIV incidence was $2.2 \%$ per year, and the estimate of the net reproduction rate of the epidemic $\left(R_{0}\right)$ reached 3.6 between 1994 and 2004 (i.e., 3.6 newly infected persons between 1994 and 2004 per person infected in 1994). Mean HIV incidence was almost equal for the three groups considered (i.e., high, medium and low levels of circumcision): $2 \%$ mean incidence per year in provinces with a high level of circumcision (specifically, $2.3 \%$ in the Eastern Cape; $1.6 \%$ in Limpopo); $2.5 \%$ mean incidence per year in provinces with a medium level of circumcision (2\% in the Free State; $2.7 \%$ in Gauteng), and $2.1 \%$ mean incidence per year in provinces with a low level of circumcision $(1.4 \%$ in the Western Cape; $1.6 \%$ in the Northern Cape; $2.6 \%$ in KwaZulu-Natal, $1.9 \%$ in Mpumalanga, and $2 \%$ in the North West).

With respect to $R_{0}$, the pattern was reversed: $R_{0}$ was higher (6.4) in provinces with a high level of circumcision (6.1 and 6.8 in the Eastern Cape and Limpopo, respectively), medium $\left(R_{0}=4.5\right)$ in provinces with a medium level of circumcision (5.3 and 2.6 in Gauteng and the Free State, respectively), and lower $\left(R_{0}=2.7\right)$ in provinces with a low level of circumcision (2.2 in KwaZulu-Natal; 2.0 in Mpumalanga; 3.4 in the North West). Moreover, it should be noted that the estimate of the net reproduction rate of the epidemic was very high for the Western Cape $\left(R_{0}=14.5\right)$ and the Northern Cape $\left(R_{0}=9.1\right)$; these two provinces have a somewhat different social fabric and large portion of the population who belong to the 'coloured' racial group. More importantly, South African provinces with higher values of $R_{0}$ were primarily areas with relatively low levels of HIV seroprevalence in 1994, as may have been anticipated. (Indeed, the computation of $R_{0}$ assumed that all cases of HIV transmission occurred within the given province, an obvious oversimplification in a country where internal migration is frequent and accounts for a large share of the spread of HIV.) Note that different provincial levels of HIV prevalence in 1994 may be explained by a variety of socioeconomic factors. In particular, in 1994, areas with the most cases of HIV infection were the largest industrial centres and the areas hosting the two largest harbours (Durban and Richard's Bay).

In summary, there was no evidence that HIV transmis- sion over the 1994-2004 period was slower in those South African provinces with higher levels of male circumcision.

Thirteen countries with Demographic and Health Surveys Among the thirteen Demographic and Health Surveys (DHS) investigated, those of eight countries (Burkina Faso, Cote d'lvoire, Ethiopia, Ghana, Niger, Rwanda, Tanzania and Zimbabwe) showed no significant difference in male HIV seroprevalence in circumcised and uncircumcised groups; data from two countries showed lower HIV prevalence among circumcised groups, as expected (Kenya and Uganda), and three countries (Cameroon, Lesotho and Malawi) showed higher prevalence among circumcised groups, contrary to expectations (Table 2).

The combined risk ratio $(R R)$ calculated by metaanalysis was 0.99 , not significantly different from 1 (95\% $\mathrm{Cl}=0.94-1.05)$. Therefore, for the continent as a whole, the analysis found no statistical evidence to support the belief that male circumcision reduces the level of HIV seroprevalence in a national population. Of course, there is heterogeneity among the countries investigated; below I present further case study analyses. Furthermore, in countries where a large majority of the population is either circumcised or not, minorities who have the opposite practice might also be quite different for a variety of socio-economic reasons. Two countries, Tanzania and Lesotho, deserve special mention because they represent a more balanced situation of the prevalence of circumcision (that is, large groups are either circumcised or not).

\section{Tanzania}

Tanzania has a large number of ethnic groups, with some 110 groups recorded in the Ethnologue database (see www. ethnologue.com). In this country, some groups practice male circumcision, others do not, so that about $70 \%$ of the total male population is circumcised, a much higher proportion than in South Africa. Roughly speaking, groups living in western regions of Tanzania and closer to Lake Victoria do not practice male circumcision, whereas groups living in eastern regions of the country often practice circumcision.

The pattern of relationship between male circumcision and HIV infection in Tanzania differed from what was expected. The 2003 DHS for Tanzania showed higher HIV seroprevalence among circumcised men (6.5\%) than among

Table 1: Parameters of the dynamics of HIV infection in provinces of South Africa, by circumcision status, $1994-2004\left(R_{0}=\right.$ net reproduction rate of the epidemic)

\begin{tabular}{|c|c|c|c|c|}
\hline & \multicolumn{4}{|c|}{ Level of circumcision ${ }^{1}$} \\
\hline & High & Medium & Low & Total \\
\hline Number of provinces & 2 & 2 & 5 & 9 \\
\hline Population (2001), millions & 11.7 & 11.5 & 21.6 & 44.8 \\
\hline Population increase $1994-2004^{2}$ & 1.18 & 1.20 & 1.14 & 1.17 \\
\hline HIV seroprevalence in $1994(\%)^{3}$ & 3.9 & 7.1 & 9.5 & 7.5 \\
\hline HIV seroprevalence in 2004 (\%) & 24.1 & 32.3 & 30.7 & 29.5 \\
\hline Mean yearly HIV incidence (\%) & 2.0 & 2.5 & 2.1 & 2.2 \\
\hline Estimate of $R_{0}$ & 6.4 & 4.5 & 2.7 & 3.6 \\
\hline
\end{tabular}

${ }_{1}^{1}$ Levels of circumcision — High level: Eastern Cape, Limpopo; Medium level: Gauteng, Free State; Low level: KwaZulu-Natal, Mpumalanga, North West, Northern Cape, Western Cape

${ }^{2}$ Population increase refers to the female population aged 15-49

${ }^{3}$ HIV seroprevalence among pregnant women who attended antenatal clinics 
Table 2: HIV prevalence in the male adult population according to circumcision status; data are from demographic and health surveys (DHS) conducted in 2003-2006 (see separate references listing); RR = risk ratio; 95\% Cl = 95\% confidence interval; NS = no significant difference in prevalence between groups ( $R R<>1, p>0.05)$; denotes higher prevalence for circumcised groups $(\mathrm{RR}>1, p<0.05) ;-$ denotes lower prevalence for circumcised groups $(\mathrm{RR}<1, p<0.05)$

\begin{tabular}{|c|c|c|c|c|c|c|c|}
\hline & \multicolumn{3}{|c|}{$\%$ HIV-positive } & \multicolumn{3}{|c|}{$\begin{array}{l}\text { Comparison of the two groups } \\
\qquad 95 \% \mathrm{Cl}\end{array}$} & \multirow[b]{2}{*}{ Signif. } \\
\hline & $\begin{array}{c}\text { Percent } \\
\text { circumcised }\end{array}$ & Circumcised & $\begin{array}{c}\text { Not } \\
\text { circumcised }\end{array}$ & $\mathrm{RR}$ & Min. & Max. & \\
\hline Burkina Faso & 89.7 & 1.8 & 2.9 & 0.62 & 0.328 & 1.174 & NS \\
\hline Cameroon & 91.8 & 4.1 & 1.1 & 3.73 & 1.369 & 10.150 & + \\
\hline Cote d'Ivoire & 96 & 2.8 & 3.8 & 0.74 & 0.32 & 1.71 & NS \\
\hline Ethiopia & 92.3 & 0.9 & 1.1 & 0.82 & 0.309 & 2.165 & NS \\
\hline Ghana & 95.3 & 1.6 & 1.4 & 1.14 & 0.356 & 3.673 & NS \\
\hline Kenya & 83.4 & 3 & 12.6 & 0.24 & 0.171 & 0.332 & - \\
\hline Lesotho & 48.6 & 22.8 & 15.2 & 1.50 & 1.236 & 1.820 & + \\
\hline Malawi & 20.7 & 13.2 & 9.5 & 1.39 & 1.050 & 1.839 & + \\
\hline Niger & 99.5 & 1 & 0 & & & & NS \\
\hline Rwanda & 11.1 & 3.5 & 2.1 & 1.67 & 0.975 & 2.848 & NS \\
\hline Tanzania & 69.7 & 6.5 & 5.6 & 1.16 & 0.905 & 1.489 & NS \\
\hline Uganda & 24.9 & 3.8 & 5.6 & 0.68 & 0.531 & 0.868 & - \\
\hline Zimbabwe & 10.5 & 16.6 & 14.2 & 1.17 & 0.95 & 1.44 & NS \\
\hline Total & & & & 0.99 & 0.94 & 1.05 & NS \\
\hline
\end{tabular}

uncircumcised men (5.6\%). This was likely partly due to a correlation with urbanisation, a major factor in determining HIV prevalence. However, even after controlling for urbanisation, no correlation between male circumcision and HIV seroprevalence was found: $9.5 \%$ versus $9.7 \%$ prevalence for circumcised versus uncircumcised groups, respectively, in urban areas, and $4.6 \%$ versus $5.2 \%$ for those groups in rural areas, with neither of the differences significant ( $p=0.938$ and $p=0.425$, respectively).

Further investigation using multivariate methods confirmed the individual risk of contracting HIV, according to circumcision status. Using the data from the same DHS survey, a series of linear logistic regression analyses were performed, using age, age-square, urbanisation, wealth, and circumcision status as predictors of HIV seroprevalence for males. Results showed an odds ratio of 0.66 associated with male circumcision $(95 \% \mathrm{Cl}=0.49-0.88)$, again consistent with other findings. This, however, does not change the key finding - that HIV seroprevalence estimates after 25 years of transmission opportunity were similar for circumcised and uncircumcised groups of males, even after controlling for urbanisation.

\section{Lesotho}

Lesotho is a much more homogenous country, with a single ethnic group, low level of urbanisation (about 28\%) and relatively high level of education compared to other African countries. HIV seroprevalence is also fairly homogenous throughout the country, with only minor differences revealed in relation to urbanisation, ecological zone, district, level of education and wealth. The country's population is essentially Christian, with various churches operating. Here again, contrary to expectations, HIV seroprevalence was significantly higher among circumcised men than among uncircumcised men, aged $15-59$ (22.8\% versus $15.2 \%$ prevalence, respectively), and this was true in relation to any socio-economic characteristics considered in the DHS final report. Therefore, the difference in prevalence between the two groups could not be explained by any single socio-economic confounder, with the exception of urbanisation (urban areas had higher HIV prevalence and lower proportions of circumcised men). In a multivariate analysis of the risk factors of HIV seroprevalence, male circumcision was no longer found to be a risk factor $(\mathrm{OR}=1.00$, $95 \% \mathrm{Cl}=0.79-1.27$ ), after controlling for age, age-square, place of residence, ecological zone, level of education, wealth and religion.

\section{Other countries}

Other countries with small proportions of uncircumcised men provide additional evidence, although they are more difficult to interpret because of the high selectivity of groups with different circumcision practices.

In Burkina Faso (2003 DHS), where $90 \%$ of men are circumcised, the difference of HIV seroprevalence between circumcised and uncircumcised groups was not significant $(p=0.142)$. Furthermore, the capital city Ouagadougou had among regions in that country the highest level of circumcision (97\%), but also the highest male HIV seroprevalence $(3.9 \%)$. In the eastern province of Burkina Faso, where almost every male is circumcised (99.1\%), male HIV seroprevalence was also above the national average $(3.1 \%)$. Two ethnic groups that live in the southern part of the country had a low level of circumcision, the Gurunsi and the Lobi: they had higher male HIV seroprevalence than average $(4.2 \%)$, but not significantly different from that of two other neighbouring ethnic groups also living in the south, the Dioula and the Bissa (3.8\% prevalence), who are almost all circumcised ( $97 \%$ circumcised).

In Cameroon (2004 DHS), where $92 \%$ of men are circumcised, the tiny groups of uncircumcised men had lower HIV seroprevalence than others $(1.1 \%$ versus $4.1 \%$ prevalence, respectively), the difference being significant ( $p=0.010)$.

In Ethiopia (2005 DHS), the prevalence of HIV among men was low (1\%), and most men were circumcised $(92 \%)$, so that any differential analysis was virtually impossible. 
In Ghana (2003 DHS), where 95\% of men are circumcised, there was no difference in male HIV seroprevalence between the two groups $(1.6 \%$ versus $1.4 \%$ prevalence for circumcised and uncircumcised groups, respectively; $p=0.823$ ), and the numbers were too small for pursuing the analysis by ethnicity.

In Malawi (2004 DHS), a minority of men are circumcised $(21 \%)$. Plotted according to ethnicity, the correlation between circumcision and male HIV seroprevalence was positive $(r=0.404)$, meaning that circumcised groups tended to be more HIV-infected than the uncircumcised groups. The group practicing almost universal circumcision (the Yao) was more infected than the national average (12.4\% prevalence), as was the case for the Lomwe $(30 \%$ circumcised; 18\% HIV-positive), whereas groups hardly practicing circumcision (the Chewa, Tumbuka and Nkonde) had lower values of male HIV prevalence $(5.7 \%, 5.1 \%$ and $4.9 \%$, respectively). The Ngoni were a counter-example, practicing a low level of circumcision (4.2\%) and having higher male HIV seroprevalence (14.5\%). These correlations could not be explained by interactions with any known socio-economic factor, although, notably, higher education was negatively related with circumcision and positively related with HIV prevalence, probably for independent reasons. In a multivariate analysis of the risk factors of HIV seroprevalence, male circumcision was no longer found to be a risk factor $(\mathrm{OR}=1.38,95 \% \mathrm{Cl}=0.97-1.96)$, after controlling for age, age-square, place of residence, level of education, wealth and religion. The ethnic correlations appeared primarily the product of a regional pattern: males in southern Malawi were more often circumcised (33.1\%) and more often HIV-infected (15.1\% prevalence); in northern Malawi, males were less often circumcised (5\%) and less often HIV-infected (5.4\% prevalence). These findings show the complexity of the relationship between circumcision and HIV prevalence. Numerous reasons may be evoked for understanding the Malawi pattern: the south is more urbanised, poorer, and tends to send more migrant workers abroad. A recent study (based on small numbers and not employing proper sampling) indicates that southern populations in Malawi are more prone to partnermobility, to late marriage and to divorce than are northern populations (Obare, 2007). In any case, and whatever the ultimate determinants in Malawi, male circumcision did not protect the southern populations from acquiring HIV in high numbers.

In Niger (2006 DHS), where virtually all men are circumcised, there was no case of HIV infection reported among the tiny minority of males who were not circumcised.

In Rwanda (2005 DHS), the prevalence of circumcision and that of HIV were both too low to permit a differential analysis. Note, however, that Rwanda has kept low levels of HIV prevalence despite low levels of circumcision (11\%), and despite the fact that the country is located near the epicentre of the HIV epidemic.

In Kenya (2003 DHS), data on individual risk and population effects were consistent with expectations. The only sizeable minority not practicing routine circumcision, the Luo (about $12 \%$ of the population) who live near Lake Victoria, had higher male HIV seroprevalence than other groups $(17.5 \%$ versus $2.8 \%$ prevalence; $p<0.001)$, and even among the Luo the few circumcised men were less often HIV-infected than were uncircumcised men, although the difference was not significant (about $10 \%$ versus $20 \%$ HIV prevalence among circumcised versus uncircumcised Luo men, respectively; $p=0.091$ ).

Similarly, in Uganda (2005 AIS), where only a minority of men are circumcised, the correlation among the ethnic groups between prevalence of circumcision and HIV was negative, but remained small $(r=-0.203)$. The pattern according to ethnicity was complex. The only group widely practicing circumcision ( $80 \%$ of Bagisu/Sabiny males circumcised) had lower than average HIV seroprevalence $(3.5 \%)$. Two other groups also practicing circumcision, although at a much lower level (the Baganda and Basoga, $31 \%$ and $35 \%$ of males circumcised, respectively), had on the contrary higher than average HIV seroprevalence (5.8\% and $5.6 \%$, respectively). The highest seroprevalence $(12.8 \%)$ was found in a group practicing circumcision to a certain extent ( $22 \%$ of Batoro males circumcised), whereas the lowest seroprevalence $(1.1 \%)$ was found in a group (the Karimojong) who was not practicing circumcision. Furthermore, circumcision was more prevalent in urban areas than in rural areas of Uganda (36\% urban versus $23 \%$ rural males circumcised); HIV infection was more prevalent in urban areas as well $(6.7 \%$ urban versus $4.7 \%$ rural HIV prevalence), a pattern opposite from that expected. So, even if the correlation among ethnic groups was somewhat negative in Uganda, the pattern was by no means straightforward.

\section{Discussion}

The demographic evidence indicates that the relationship between male circumcision and HIV seroprevalence is complex, and that both positive and negative relations can be found for a variety of reasons. No evidence of an overall protective effect of male circumcision was found for the countries studied, and if there is an effect at the individual level it is buried in a mix of many other intervening factors.

One might argue that the 'natural experiment' in South Africa is not identical to systematic voluntary circumcision. However, if circumcision were to be recommended, it is unlikely to become universal, so that the natural experiment is probably close to a real-world situation. Surveys about males' intention to be circumcised have shown that about two-thirds of men expressed an interest in the practice (Westercamp \& Bailey, 2007), so that the natural situation of groups practicing circumcision is probably close to what could happen if programmes of voluntary circumcision were successful in the country.

The situation in Tanzania also seems close to an experimental situation. One could argue that the two groups circumcised and uncircumcised males - are not exposed to the virus the same way. However, since circumcised groups typically live farther away from Lake Victoria, they may be less exposed or later exposed to HIV (which reinforces the main argument). One exception so far, in Tanzania, is the Zanzibar Islands, which have low HIV prevalence. The populations on these islands are highly conservative Muslims, and they are fairly isolated from the continent's mainland, so their exposure to HIV is likely different from 
populations in the rest of the country.

The most puzzling finding from the study concerns Lesotho, where HIV seroprevalence was found consistently higher among circumcised men, despite otherwise high homogeneity of the country's population. The case of this country, perhaps typical of southern African countries with high levels of HIV seroprevalence, clearly shows that little may be expected from voluntary circumcision programmes in advanced generalised epidemics.

Population-effects over long periods of time are not necessarily identical with measures of individual risk for shorter periods. Indeed, under intense and repeated exposure, a $50 \%$ lower annual risk does not translate into a large reduction at the population level. A good example is provided by contraception. A $50 \%$ efficacious contraceptive method, such as periodic abstinence (the rhythm method), does not protect against pregnancy in the long run: certainly, it has an effect on the length of birth intervals and mean-age at birth stratified by birth order, but not on a woman's lifetime probability of getting pregnant.

Similarly, comparisons with vaccines are worth mentioning and many contrasting situations have been documented in this field. An example of equivalence between individualeffects and population-effects is provided by the measles vaccine: its efficacy (about $96 \%$ in clinical trials) translates readily into population-effect. Mass vaccination stops measles epidemics for several years, and has a strong impact on incidence of the disease. An example of low population-effect, with a $50 \%$ efficacy in clinical trials, is provided by the cholera vaccine. This vaccine protects only for a short period of time in about half the cases, does not have much effect on cholera epidemics, and is currently not even recommended for mass usage, even though its effect on herd immunity clearly appears when proper multivariate analysis is conducted (as was often the case for male circumcision in this study) (Ali, Emch, Von Seidlein, Yunus, Scoak, Rao et al., 2005). A third example of populationeffect that is higher than that expected from clinical trials is given by the vaccine against Bordetella pertussis (whooping cough). In clinical trials this vaccine has shown low efficacy to protect against infection, though it has a much higher efficacy for preventing severe disease and death. Despite its low efficacy in clinical trials, the whooping cough vaccine has a major population-effect, and can stop an epidemic in a way similar to the measles vaccine. The British health authorities who stopped vaccinations against whooping cough in the 1970 s quickly learned that this led to a return of large epidemics, so that they had to reinstall the vaccination policy (Pollard, 1980). These three examples show that there is not always a one-to-one relationship between findings from clinical trials and the dynamics of communicable diseases in large populations. The case of HIV is further complicated by sexual transmission modes, and hence the possibility of different dynamics among men and women. To return to the original argument proposed by Fink (1987), epidemics of syphilis were at least as severe in the United States, where most men were circumcised, as in Western Europe, where more often they were not circumcised. Likewise, heterosexual transmission of HIV never became widespread in Europe, as it is the case in the United States. In his comprehensive review on the topic, Van Howe
(1999, p. 61) concluded that "the medical literature does not support the theory that circumcision prevents STDs." Similarly, the Cochrane Library review on the effect of male circumcision on HIV prevalence found "insufficient evidence to support an effect of male circumcision on HIV acquisition in heterosexual men" (Siegfried, Muller, Volmink, Deeks, Egger, Low et al., 2003).

Some authors have questioned the quality of data on circumcision reported in Demographic and Health Surveys and the quality of the circumcision itself when done by traditional practitioners. However, the reported effect of male circumcision in clinical trials is basically the same as in observational studies, and also in general populations after controlling for confounding factors, which reinforces the value of the demographic information.

Decisions of whether or not to circumcise for nontherapeutic reasons remains controversial in situations of high HIV incidence. On one hand, any intervention that can reduce HIV-infection risk seems desirable; on the other hand, it does not seem likely that circumcising a large proportion of the male population will have a large populationeffect. Furthermore, universal circumcision would require massive involvement of health personnel, already in short supply in many African countries. Even in South Africa, a fairly developed country, physicians and nurses are scarce and their numbers are not large enough to manage antiretroviral therapy for all who need it. The 2006 World Health Report (WHO, 2006) puts strong emphasis on health personnel, their importance for developing countries, and their shortage throughout sub-Saharan Africa. In contrast, alternative strategies do exist for controlling the spread of HIV, such as the well-known A-B-C strategy (abstain, be faithful, use condoms); these may be less costly and are known to be efficient at the population level. For instance, the dynamics of the HIV epidemic in Thailand were changed with massive use of condoms; in Uganda a major change was obtained primarily by changing sexual behaviour; even in South Africa, the incidence of the disease, given the size of the HIV-infected population, has decreased since 2001, most likely as the result of increased condom use (documented in HSRC surveys) and some changes in sexual behaviour.

Given what is known at the individual level, one would have expected HIV incidence or prevalence in circumcised groups of men to be consistently about $20 \%$ lower than in uncircumcised groups. This was not the case according to the results of this study. Until this discrepancy between demographic evidence and expectations from epidemiological evidence is resolved, is it wise to recommend mass circumcision? Also, the implementation of mass circumcision raises serious ethical concerns, well documented elsewhere (Clark, 2006).

Once more, the dynamics of generalised HIV epidemics in Africa appear more complex than originally thought. Male circumcision appears only as a minor factor amid many others contributing to the spread of HIV, such as the complex web of social, cultural and economic interactions surrounding sexual behaviour, especially among adolescents and young adults. These complex relationships merit further field studies - as well as investigations through mathematical modelling. 
Acknowledgements - The author warmly thanks all those who shared ideas, comments and data for this study, in particular Paul Pronyk, Slim Abdool Karim, Rob Dorrington, Pauline Leclerc, Muriel Vray and Arnaud Fontanet.

The author - Michel Garenne (PhD, demography) is Director of Research at the French Institute for Research and Development (IRD) and is currently working at the Pasteur Institute, Emerging Diseases Unit, in Paris. He is also honorary Associate Professor at the University of the Witwatersrand, Johannesburg. He directed the Niakhar Demographic Surveillance System in Senegal in the 1980s and has collaborated with the Agincourt Health and Demographic Surveillance System in South Africa since 1992. He is author of numerous publications on population and health issues in Africa, and has taught demography at several universities in Europe (Paris, Clermont-Ferrand, Heidelberg, Antwerp) and in the United States (Harvard).

\section{Demographic and Health Surveys (DHS)}

Burkina Faso - Institut National de la Statistique et de la Démographie (INSD) \& ORC Macro (2004) Enquête Démographique et de Santé du Burkina Faso 2003. Calverton, Maryland, INSD and ORC Macro.

Cameroon - Institut National de la Statistique (INS) \& ORC Macro (2004) Enquête Démographique et de Santé du Cameroun 2004. Calverton, Maryland, INS and ORC Macro.

Cote d'Ivoire - Institut National de la Statistique (INS), Ministère de la Lutte contre le Sida [Côte d'Ivoire] \& ORC Macro (2006) Enquête sur les Indicateurs du Sida, Côte d'Ivoire 2005. Calverton, Maryland, INS and ORC Macro.

Ethiopia - Central Statistical Agency (CSA) [Ethiopia] \& ORC Macro (2006) Ethiopia Demographic and Health Survey 2005. Addis Ababa, Ethiopia and Calverton, Maryland, CSA and ORC Macro.

Ghana - Ghana Statistical Service (GSS), Noguchi Memorial Institute for Medical Research (NMIMR) \& ORC Macro (2004) Ghana Demographic and Health Survey 2003. Calverton, Maryland, GSS, NMIMR and ORC Macro.

Kenya - Central Bureau of Statistics (CBS) [Kenya], Ministry of Health (MoH) [Kenya] \& ORC Macro (2004) Kenya Demographic and Health Survey 2003. Calverton, Maryland, CBS, MoH and ORC Macro.

Lesotho - Ministry of Health and Social Welfare (MoHSW) [Lesotho], Bureau of Statistics (BOS) [Lesotho] \& ORC Macro (2005) Lesotho Demographic and Health Survey 2004. Calverton, Maryland, $\mathrm{MoH}, \mathrm{BOS}$ and ORC Macro.

Malawi - National Statistical Office (NSO) [Malawi] \& ORC Macro (2005) Malawi Demographic and Health Survey 2004. Calverton, Maryland, NSO and ORC Macro.

Niger - Institut National de la Statistique (INS) \& Macro International Inc. (2007) Enquête Démographique et de Santé et à Indicateurs Multiples du Niger 2006. Calverton, Maryland, INS and Macro International Inc.

Rwanda - Institut National de la Statistique du Rwanda (INSR) \& ORC Macro (2006) Rwanda Demographic and Health Survey 2005. Calverton, Maryland, INSR and ORC Macro.

Tanzania - Tanzania Commission for AIDS (TACAIDS), National Bureau of Statistics (NBS) \& ORC Macro (2005) Tanzania HIV/ AIDS Indicator Survey 2003-04. Calverton, Maryland, TACAIDS, NBS and ORC Macro.

Uganda - Ministry of Health (MoH) [Uganda] \& ORC Macro (2006) Uganda HIVIAIDS Sero-Behavioural Survey 2004-2005. Calverton, Maryland, MoH and ORC Macro.

Zimbabwe - Central Statistical Office (CSO) [Zimbabwe] \& Macro International Inc. (2007) Zimbabwe Demographic and Health Survey 2005-06. Calverton, Maryland, CSO and Macro International Inc.

\section{References}

Ali, M., Emch, M., Von Seidlein, L., Yunus, M., Scoak, D.A., Rao, M., Hilmgren, J. \& Clemens, J.D. (2005) Herd immunity conferred by killed oral cholera vaccines in Bangladesh: a re-analysis. The Lancet 366, pp. 44-49.

Auvert, B., Taljaard, D., Lagarde, E., Sobngwi-Tambekou, J., Sitta, R. \& Puren, A. (2005) Randomized, controlled intervention trial of male circumcision for reduction of HIV-infection risk: the ANRS 1265 trial. PLoS Medicine 2(11), pp. 1-111.

Bailey, R.C., Moses, S., Parker, C.B., Agot, K., MacLean, I., Krieger, J.N., Willams, C.F.M., Campbell, R.T. \& Nidnya-Achola, J.O. (2007) Male circumcision for HIV prevention in young men in Kisumu, Kenya: a randomised controlled trial. The Lancet 369, pp. 643-656.

Bongaarts, J., Reining, P., Way, P. \& Conant, F. (1989) The relationship between male circumcision and HIV infection in African populations. AIDS 3(6), pp. 373-377.

Clark, P.A. (2006) To circumcise or not to circumcise? Health Progress 87(5), pp. 1-9.

Fink, A. (1986) A possible explanation for heterosexual male infection with AIDS. New England Journal of Medicine 315(8), p. 1167.

Fink, A. (1987) Circumcision and heterosexual transmission of HIV infection to men. New England Journal of Medicine 316, pp. 1546-1547.

Gray, R.H., Kigozi, G., Serwadda, D., Makumbi, F., Watya, S., Nalugoda, F., Kiwanuka, N., Moulton, L.H., Chaudhary, M.A., Chen, M.Z., Sewankambo, N.K., Wabwire-Mangen, F., Bacon, M.C., Williams, C.F.M., Opendi, P., Reynolds, S.J., Laeyendecker, O., Quinn, T.C. \& Waver, M.J. (2007) Male circumcision for HIV prevention in men in Rakai, Uganda: a randomised controlled trial. The Lancet 369, pp. 657-666.

Gray, R.H., Li, X., Wawer, M.J., Gange, S.J., Serwadda, D., Sewankambo, N.K., Moore, R., Wabwire-Mangen, F., Lutalo, T., Quinn, T.C. \& the Rakai Project Group (2003) Stochastic simulation of the impact of antiretroviral therapy and HIV vaccines on HIV transmission; Rakai, Uganda. AIDS 17(13), pp. 1941-1951.

Human Sciences Research Council (HSRC) (2002) Nelson Mandela/HSRC Study of HIVIAIDS. South African National HIV Prevalence, Behavioural Risks and Mass Media, Household Survey 2002. Cape Town, South Africa, HSRC Publishers.

Human Sciences Research Council (HSRC) (2005) South African National HIV Prevalence, HIV Incidence, Behaviour and Communication Survey, 2005. Cape Town, South Africa, HSRC Publishers.

Moses, S., Bradley, J.E., Nagelkerke, N.J., Ronald, A.R., NdinyaAchola, J.O. \& Plummer, F.A. (1990) Geographical patterns of male circumcision practices in Africa. International Journal of Epidemiology 19(3), pp. 693-697.

Moses, S., Plummer, F.A., Bradley, J.E., Ndinya-Achola, J.O., Nagelkerke, N. \& Ronald, A.R. (1994) The association between lack of male circumcision and risk for HIV infection. Sexually Transmitted Diseases 21(4), pp. 201-210.

Nagelkerke, N.J., Moses, S., De Vlas, S.J. \& Bailey, R.C. (2007) Modelling the public health impact of male circumcision for HIV prevention in high prevalence areas in Africa. BMC Infectious Diseases 7, p. 16. Available online: http://www.pubmedcentral. nih.gov/articlerender.fcgi?artid=1832203.

Obare, F. (2007) An assessment of the HIV risk factors and variations in HIV prevalence in rural Malawi. Paper presented at the Fifth African Population Conference, Arusha, Tanzania, 10-14 December 2007.

Orroth, K.K., Freeman, E.E., Bakker, R., Buvé, A., Glynn, J.R., Boily, M.C., White, R.G., Habbema, J.D.F. \& Hayes, R.J. (2007) Understanding the differences between contrasting HIV epidemics in east and west Africa: results from a simulation model of the 
Four Cities Study. Sexually Transmitted Infections 83 (supplement 1), pp. i5-i16.

Pollard, R. (1980) Relation between vaccination and notification rates for whooping cough in England and Wales. The Lancet 1(8179), pp. 1180-1182.

RADAR (Rural AIDS and Development Action Research Programme) (2002) Social interventions for HIVIAIDS: intervention with micro-finance for AIDS and gender equity. IMAGE Study, Evaluation Monograph No 1.

Siegfried, N., Muller, M., Volmink, J., Deeks, J., Egger, M., Low, N., Weiss, H., Walker, S. \& Williamson, P. (2003) Male circumcision for prevention of heterosexual acquisition of HIV in men (Cochrane Review). In: The Cochrane Library, Issue 3, 2003. Oxford, Update Software.

Van Howe, R.S. (1999) Does circumcision influence sexually transmitted diseases? A literature review. BJU International 83(supplement 1), pp. 52-62.
Weiss, H.A., Quigley, M.A. \& Hayes, R.K. (2000) Male circumcision and risk of HIV infection in sub-Saharan Africa: a systematic review and meta-analysis. AIDS 174, pp. 2361-2370.

Westercamp, N. \& Bailey, R.C. (2007) Acceptability of male circumcision for prevention of HIVIAIDS in sub-Saharan Africa: a review. AIDS and Behavior 11(3), pp. 341-355.

Williams, B.G., Lloyd-Smith, J.O., Gouws, E., Hankins, C., Getz, W.M., Hargrove, J., De Zoysa, I., Dye, C. \& Auvert, B. (2006) The potential impact of male circumcision on HIV in sub-Saharan Africa. PLoS Medicine 3(7), p. e262.

World Health Organization (WHO) (2006) The World Health Report 2006 - Working Together for Health. Geneva, WHO. 